\title{
Kossel diffraction and X-ray standing waves: two birds of one feather
}

Th. Gog, D. Novikov, J. Falta, A. Hille and G. Materlik

Hamburger Synchrotronstrahlungslabor HASYLAB am Deutschen Elektronen-Synchrotron DESY, 22603 Hamburg, Germany

\begin{abstract}
By virtue of the optical reciprocity theorem, Bragg-case Kossel diffraction in perfect crystals can be viewed as the reverse process of x-ray standing waves (XSW) in the sense that the location of the source of radiation and the point of detection are merely interchanged between the two. A method can then be devised to determine atomic positions in a crystal lattice by analyzing the profile of angularly resolved Kossel lines, yielding equivalent information as an analogous XSW experiment would. Technical aspects of this novel method are discussed and first experimental results involving $\mathrm{Ge}$ and $\mathrm{Er}: \mathrm{LiNbO}_{3}$ single crystal samples are presented, indicating that the equivalence between Kossel diffraction and XSW is indeed realized.
\end{abstract}

\section{Introduction and Theory}

Kossel diffracton which is diffraction of radiation from internal sources [1] arises when atoms inside a crystal are excited by some incident radiation (photons, electrons, protons, ...) and subsequently de-excite by emitting fluorescence. Most of this isotropically emitted fluorescence will be reabsorbed, scattered from other atoms or leave the crystal without interaction. Some fraction of it will be propagating in directions where Bragg's law is satisfied for a set of diffraction planes of its own lattice and for the given fluorescence energy. This fraction will then be subject to interference. Since these particular directions lie on cones with an opening half-angle of $\left(90^{\circ}-\Theta_{B}\right), \Theta_{B}$ being the Bragg angle, and central axes perpendicular to the diffraction planes (Fig. 1), patterns of light and dark "Kossel lines" may be observed on a photographic film placed near the specimen.

In the case of a dynamically diffracting crystal these Kossel lines feature a fine structure of intensity, reminiscent of the depth of the internal source and its position within the lattice [2-4]. This fine structure can be understood by realizing the inherent equivalence of XSW and Kossel diffraction through the optical reciprocity theorem. In a typical XSW experiment a plane wave, which can be considered as coming from a point source at infinity is incident on the sample under an angle in the range of a Bragg reflection. Incident and diffracted beam interfere to form a standing wave field in the region where they 


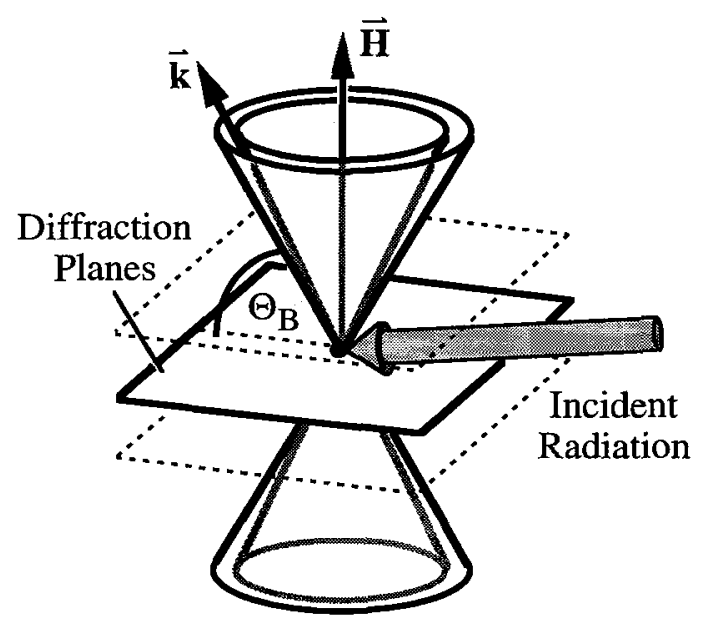

Fig. 1 Excitation of Kossel diffracted fluorescence.

overlap. Scanning through the reflection the standing wave field shifts its position, modulating the fluorescence yield from atoms in the overlap region in a way characteristic of their relative location within the diffraction plane spacing. By virtue of the reciprocity theorem, which states that "...if a source of radiation and a point of observation are interchanged, the electric field intensity will be the same in the new point of observation as it was at the old " [5], dynamical Kossel diffraction can be viewed as the reverse process of the standing wave mechanism: the source of radiation are now the fluorescing atoms inside the sample which are observed at a point far away with an angularly resolving detector. Fluorescence yields as a function of angle or in other words, Kossel line profiles, can then be written in analogy to XSW [6] as

$$
\mathrm{Y}(\Delta \Theta)=\sum_{\sigma, \pi}\left(1+R_{\sigma, \pi}(\Delta \Theta)+2 F_{c} P_{\sigma, \pi} \sqrt{R_{\sigma, \pi}(\Delta \Theta)} \cos \left(v_{\sigma, \pi}(\Delta \Theta)-\Phi_{c}\right)\right) Z_{\sigma, \pi}(\Delta \Theta)
$$

where the summation is over the two directions of polarization and the term $Z_{\sigma, \pi}(\Delta \Theta)$ is an effective thickness including absorption and extinction effects. In much the same way as in XSW, measurements of Kossel line profiles permit extraction of coherent positions $\Phi_{c}$ and coherent fractions $F_{c}$, which describe the location of the atom within the lattice plane spacing and the atomic distribution around this location.

\section{Experimental Set-Up}

The experimental set-up for this novel method, as it was used for Kossel diffraction measurements on bulk $\mathrm{Ge}(111)$ and Er-diffused $\mathrm{LiNbO}_{3}(0,0, \overline{6})$ at the undulator beamline BW1 of HASYLAB is shown in Fig. 2. A "pink" undulator $x$-ray beam, unmonochromatized and doubly reflected by a planar and toroidal mirror, impinges on the sample under such an angle that radiation of an energy slightly different from the energy of the Kossel fluorescence is Bragg-diffracted and registered in an ion chamber after passing the analyzer crystal. Monitoring the angular position of the reflection curve while repeatedly 
rocking the analyzer crystal through the range of total reflection and dynamically correcting this position, the relative orientation of sample and analyzer can be kept stable over long periods of time. This is essential for measurements of low-concentration constituents in a sample such as was the case for the Er-diffused $\mathrm{LiNbO}_{3}$ crystal. For the Kossel diffracted fluorescence the combination of analyzer and $\mathrm{Si}(\mathrm{Li})$ detector serves as an angularly resolving, energy dispersive detector yielding the Kossel line profiles, convoluted with the analyzer transfer function.

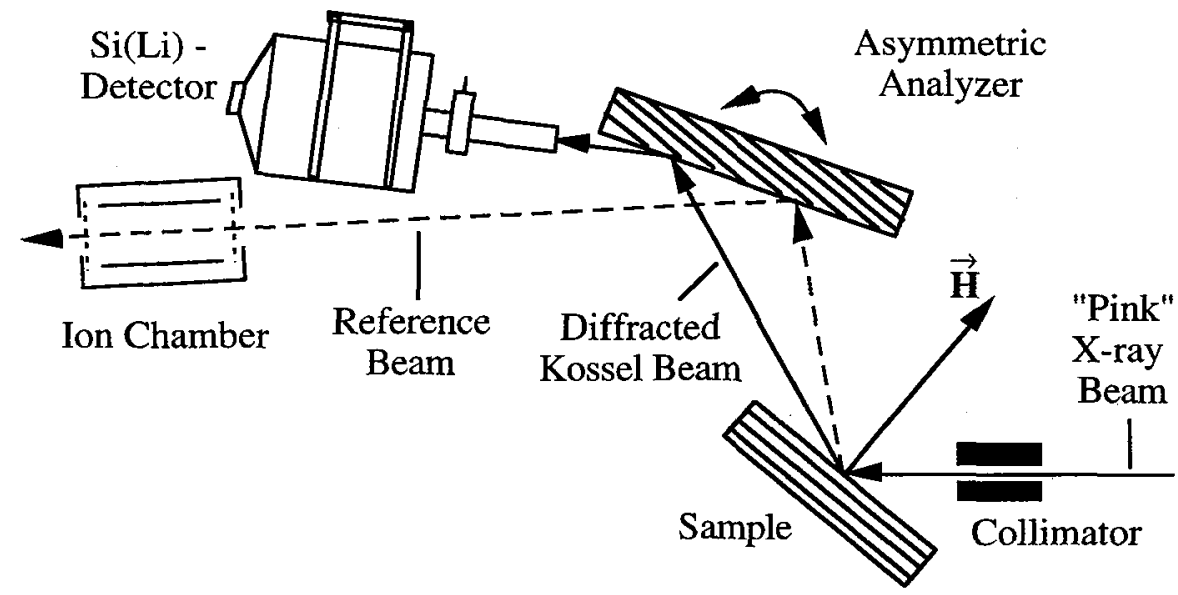

Fig. 2 Experimental configuration for the Kossel diffraction measurements. The incident and Bragg diffracted reference beam used to stabilize the relative sample / analyzer orientation is marked by dashed arrows. Kossel diffracted fluorescence, indicated by solid arrows, emerges from the sample, is reflected by the analyzer and recorded by a $\mathrm{Si}(\mathrm{Li})$ detector.

\section{Measurements with $\mathrm{Ge}(111)$ and $\mathrm{Er}: \mathrm{LiNbO}_{3}(0,0, \overline{\mathbf{6}})$}

Three examples of measured Kossel line profiles for bulk $\mathrm{Ge}(111)$ and Er-diffused $\mathrm{LiNbO}_{3}$ at a dopant concentration of about $3.6 \times 10^{19} \mathrm{~cm}^{-3}$ are shown in Fig. 3, the insets indicating the sample $/$ analyzer combination. Rocking curves provided by the reference beam were used to determine the angular region covered in the measurements, data were then fitted to the theoretical line profile given in equation (1). Extracted coherent positions and coherent fractions of $\Phi_{\mathrm{Ge}(1,1,1)}=0.97, \mathrm{~F}_{\mathrm{Ge}(1,1,1)}=0.92$, $\Phi_{\mathrm{Er},(0,0, \overline{6})}=0.54$ and $\Phi_{\mathrm{Er},(0,0, \overline{6})}=0.62$ are fully consistent with XSW measurements performed previously on the same samples.

\section{Conclusions}

The measurements presented above corroborate the claim of the inherent equivalence of Kossel diffraction and XSW and show, that even studying samples with very dilute dopants is feasible with this novel method. The simultaneous availability of numerous Kossel lines with the potential to gain full three-dimensional information about atomic positions bears an added attraction for this novel method, although mismatches in diffraction plane spacings between sample and analyzer still have to be taken into account as well as "dispersive" effects that might appear due to recording conical Kossel lines with a planar analyzer crystal. 


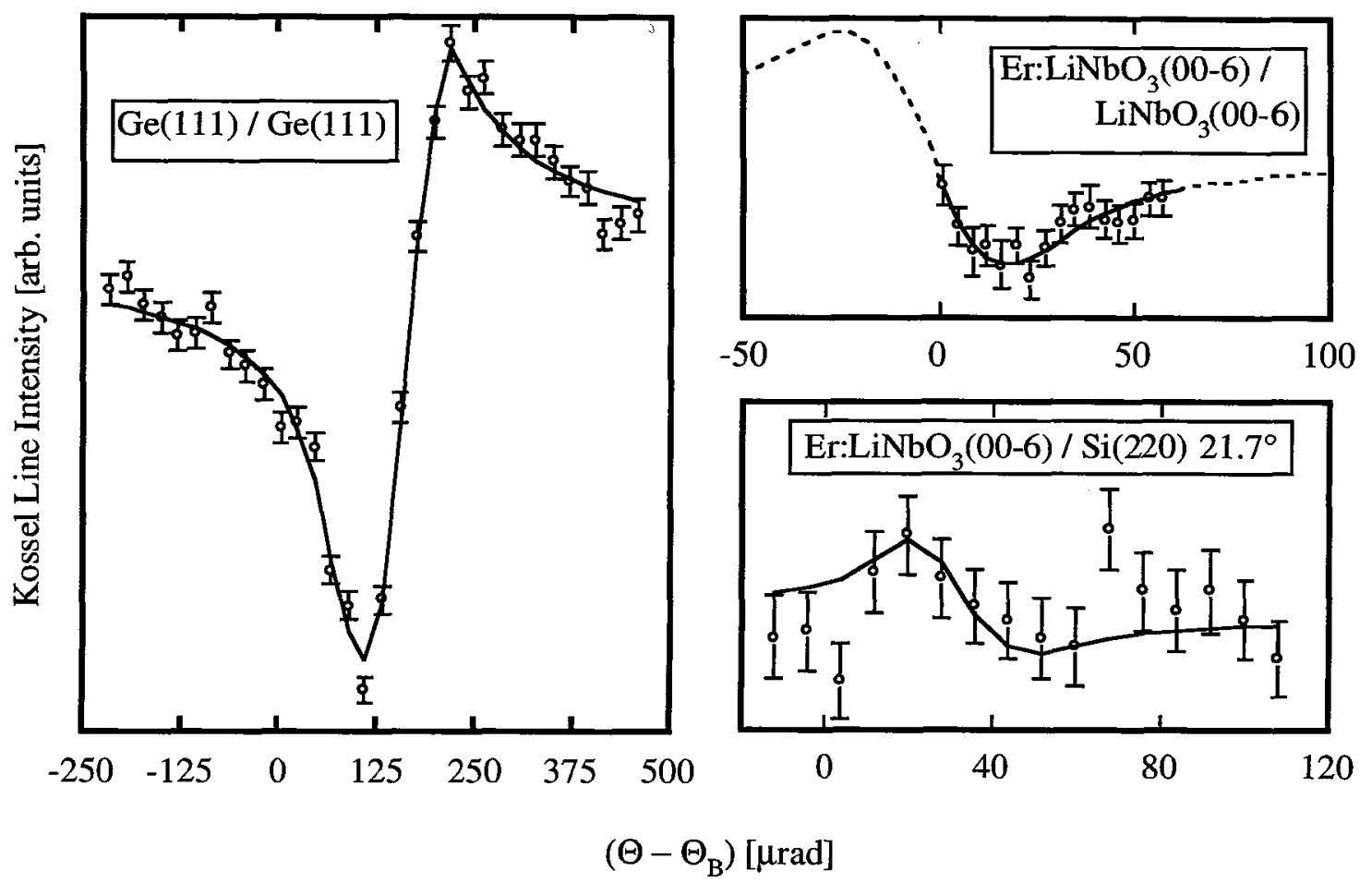

Fig. 3 Measured Kossel line profiles (markers), along with theoretical fits (solid lines) based on the dynamical theory of $x$-ray diffraction as represented in equation (1). Insets give the sample / analyzer combination. All measured profiles are consistent with fluorescence yields from conventional XSW experiments performed on the same samples.

\section{References}

[1] W. Kossel, Ergebn. exakt. Naturwiss. 16 (1937) 295

[2] M. v. Laue, Röntgenstrahlinterferenzen (Akademische Verlagsgesellschaft, Frankfurt, Germany (1960))

[3] J. T. Hutton, G. T. Trammell, J. P. Hannon, Phys. Rev. B31 (1985) 743

[4] T. Takahashi, M. Takahasi, Jpn. J. Appl. Phys. 32 (1993) 5159

[5] R. W. James, The Optical Principles of the Diffraction of X-Rays (Ox Bow Press, Woodbridge, 1982) p. 439

[6] M. J. Bedzyk, G. Materlik, Phys. Rev. B32 (1986) 6456 